\title{
Migración y ecoturismo en la Reserva de la Biosfera de Los Tuxtlas (México)
}

\author{
Ángeles Piñar Álvarez \\ Martha Elena Nava Tablada ${ }^{i}$ \\ Diana Karina Viñas Oliva ${ }^{\text {ii }}$
}

El Colegio de Veracruz (México)

\begin{abstract}
Resumen: Ante el deterioro de los recursos naturales, en 1988 es declarada la Reserva de la Biosfera de los Tuxtlas (RBT) en Veracruz, México, la cual incluye municipios con alto índice de pobreza donde los pobladores al restringir su actividad agropecuaria han tenido que emigrar hacia Estados Unidos. Paralelamente, dada la riqueza natural en la RBT, se han desarrollado iniciativas ecoturísticas amigables con el ambiente. El objetivo de investigación fue analizar la relación entre migración y ecoturismo en tres comunidades de la RBT. Se encontró que las empresas ecoturísticas comunitarias y privadas pueden proporcionar a los pobladores una actividad económica rentable para fincar proyectos de vida que los arraiguen a la comunidad y disminuyan la necesidad de emigrar por motivos económicos.
\end{abstract}

Palabras clave: Migración; Ecoturismo; Reserva de la Biosfera de Los Tuxtlas.

Title: Migration and ecotourism in the Biosphere Reserve of Los Tuxtlas (Mexico)

\begin{abstract}
In 1988, in response to the deteriorating state of its natural resources, the Los Tuxtlas Biosphere Reserve (LTBR) is established in the Mexican state of Veracruz. Included within park boundaries are municipalities characterized by a high poverty rate and whose residents, due to restrictions placed on their traditional farming activities, have had to immigrate to the United States. At the same time, the biosphere reserve's natural wealth has inspired environmentally friendly ecotourism initiatives. The purpose of our research was to analyze the relationship between migration and ecotourism in three communities located within park boundaries. We found that private and community-operated ecotourism businesses can provide inhabitants with adequate employment which in turn strengthens their ties to the community and reduces the need for them to emigrate away for financial reasons.
\end{abstract}

Keywords: Migration; Ecotourism; Tuxtlas Biosphere Reserve.

i Dra. Ángeles Piñar Álvarez y Dra. Martha Elena Nava Tablada son Profesoras e Investigadoras en El Colegio de Veracruz. E-mail: angelespinaralvarez@gmail.com y menavata@yahoo.com.mx.

ii Ing. Diana Karina Viñas Oliva es Maestra en Desarrollo Regional, El Colegio de Veracruz y becaria CONACYT, E-mail: ingevignas@gmail.com. Este artículo es parte de los resultados de investigación, proyecto 68277, financiado por FOMIX-CONACYT-Veracruz 


\section{Introducción}

El fenómeno migratorio internacional en México ha marcado el territorio a lo largo de décadas, sin embargo no había afectado la región sureste del país hasta hace aproximadamente 20 años. En dicha región, el estado de Veracruz pasó de ser receptor a expulsor de población, cambio que se relaciona con la apertura comercial internacional y la retirada del aparato estatal de la estructura productiva del sector agropecuario e industrial. Ello ha propiciado la salida de un gran número de trabajadores veracruzanos a otras regiones del país y Estados Unidos, con el fin de encontrar nuevas y mejores oportunidades de empleo y salarios (Nava, 2009).

Según datos del Consejo Estatal de Población, Veracruz pasó del lugar treinta en 1992, al cuarto lugar en tan sólo diez años, en la tabla nacional de estados con mayor salida de población hacia Estados Unidos (COESPO, 2002). Mientras, Chávez et al. (2007) afirman que hay evidencia de que al menos 900 localidades del estado de Veracruz tienen algún grado de migración hacia los Estados Unidos, aunque el fenómeno es particularmente significativo en 220.

Paralelamente, en México, a partir de la década de los noventa se incrementaron las actividades productivas comunitarias y privadas relacionadas con el turismo de la naturaleza (Molina 2007), especialmente en Áreas Naturales Protegidas (ANP), incluida la región de los Tuxtlas, por lo que es un fenómeno relativamente reciente y de enorme interés (SECTUR y CESTUR, 2007; SECTUR 2004). Cuando en 1998 se decreta ANP a la región comprendida por 8 municipios situados en los Tuxtlas, se limita las zonas de uso y aprovechamiento y se expropian algunas propiedades a los habitantes de las comunidades rurales que integran la región. Ante ello algunos pobladores, inician la búsqueda de alternativas económicas amigables con el medio ambiente. Así surgen los proyectos de ecoturismo, orientados a conservar los recursos naturales (González, 2008) y respaldar la educación para la sustentabilidad (CEATUX, 2009; García, 2009)

La Reserva de la Biosfera de los Tuxtlas guarda un sinfín de particularidades que han sido documentadas en numerosas publicaciones. Sin embargo, la mayoría se refiere tanto a las características biológicas, geográficas y ambientales de los municipios más conocidos de la Reserva (Guevara et al., 2006), como a la importancia de las instituciones locales públicas y privadas relacionadas con el manejo ambiental de los recursos (Paré y Lazos, 2003).

En el estado de Veracruz, compuesto por 212 municipios, destacan por las actividades relacionadas con el
Turismo de naturaleza cuatro municipios: Jalcomulco, Catemaco, San Andrés Tuxtla y Pajapan. Los tres últimos están localizados en los Tuxtlas y son pioneros en el fomento del turismo de naturaleza en las tres ramas que lo conforman, ecoturismo, turismo rural y turismo de aventura (SECTUR y CESTUR, 2007).

El presente trabajo analiza la actividad de ecoturismo en comunidades afectadas por la migración, para desentrañar la relación existente entre estos dos ámbitos de estudio, sobre todo en cuanto al impacto de las remesas en las inversiones económicas y productivas que realizan los habitantes, ya que esto afecta la creación de empleos y fuentes de ingreso regional. En este sentido, Piñar (2007) afirma que el fenómeno empresarial del turismo de naturaleza, persigue un uso sustentable y la preservación de los recursos naturales y culturales por parte de los habitantes y empresas locales y de los visitantes, a fin de promover el arraigo territorial, la diversificación de fuentes de empleo y la generación de proyectos productivos en las comunidades que proporcionen medios de vida a la población. Es decir, se puede "vivir de la conservación" siempre y cuando el sector de turismo de naturaleza se plantee como una estrategia de doble vía: conservar el capital natural y cultural de las comunidades rurales y atender el rezago socioeconómico en el que viven las economías locales (Piñar 2009). Paralelo a esta dinámica de conservación desde la actividad ecoturística, la migración en comunidades rurales es una realidad predominante en el proceso de globalización, que desempeña un papel importante en la comprensión del ámbito rural (Nava, 2007).

En este contexto el objetivo general de la presente investigación fue analizar la relación entre migración y actividad ecoturística en tres comunidades rurales de los municipios de Catemaco y San Andrés Tuxtla, situados en la Reserva de la Biosfera de los Tuxtlas. Para ello se describen las características de la migración y sus efectos generales en las comunidades rurales de Pozolapan y López Mateos (municipio de Catemaco) y Chuniapan de Abajo (municipio de San Andrés Tuxtla); se caracteriza la actividad ecoturística y se analiza la relación entre la actividad ecoturística y el flujo migratorio.

Abordaje Metodológico

El proceso metodológico tuvo un enfoque mixto dado que se combinó una encuesta (técnica cuantitativa) para captar las características de la migración y sus efectos en las comunidades rurales estudiadas y una entrevista semiestructurada (técnica cualitativa) para caracterizar la actividad ecoturística (Hernández et al., 2006).

Las comunidades seleccionadas en Catemaco fueron Pozolapan y López Mateos; mientras en el municipio de San Andrés Tuxtla, se incluyó la comunidad de Chunia- 
pan de Abajo. Se eligieron dichas comunidades rurales porque presentan diferentes características relativas a la migración y la actividad ecoturística que resultaba interesante explorar: a) Adolfo López Mateos, ha sido precursora del ecoturismo en los Tuxtlas, cuenta con una empresa ecoturística comunitaria consolidada y hubo un importante flujo migratorio en el pasado, sin embargo, actualmente éste es escaso; b) Pozolapan, cuenta con dos empresa ecoturísticas privadas y hay significativa presencia de migración; c) Chuniapan de Abajo, no posee oferta ecoturística y se observa un flujo migratorio im-

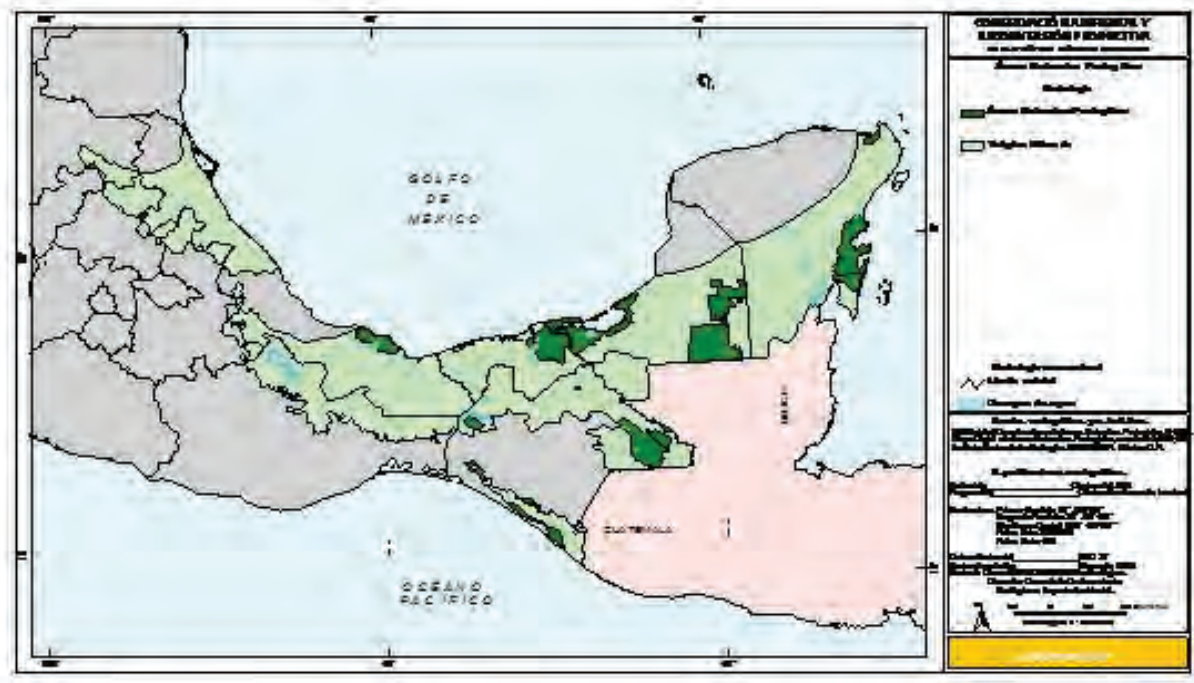

Figura 1. Areas Naturales Protegidas del Sureste Mexicano. Fuente: CONANP, 2006

La Reserva consiste de tres áreas que constituyen la zona núcleo: el Volcán San Martín Tuxtla, el Volcán Santa Martha y el Volcán San Martín Pajapan (29,720 ha), las 125,402 ha restantes integran la zona de amortiguamiento. Este conjunto de hectáreas se reparten entre los municipios de Ángel R. Cabada, Catemaco, Mecayapan, Pajapan, San Andrés Tuxtla, Santiago Tuxtla, Soteapan y Tatahuicapan de Juárez, donde despuntan numerosas iniciativas de ecoturismo (CONANP, 2006). fo López Mateos sólo se localizaron cuatro familias con migrantes activos (7 migrantes en total), por lo que no se consideró pertinente aplicar la encuesta y la información sobre la migración se captó mediante entrevistas a informantes clave. Del conjunto de empresas comunitarias y privadas de ecoturismo inventariadas por la SECTUR y CESTUR (2007), se seleccionaron tres en el municipio de Catemaco: en la comunidad de López Mateos, la empresa ecoturística comunitaria "Selva El Marinero"; y en Pozolapan, dos empresas privadas, "Bahía Escondida" y "El Teterete". En el municipio de San Andrés Tuxtla, no se realizó ninguna entrevista en Chuniapan de Abajo, porque no se identificaron empresas ecoturísticas, a pesar del interés mostrado por la población hacia esta actividad económica.

La región de los Tuxtlas como Área Natural Protegida

La región de los Tuxtlas es de gran importancia ecológica por su diversidad de especies, muchas de ellas endémicas. Por tal motivo, en 1998, fue publicado en el Diario Oficial de la Federación, el Decreto por el que se declara a esta región como Área Natural Protegida, con el carácter de Reserva de la Biosfera, con una superficie total de 155 mil 122 ha dentro del conjunto de Áreas Naturales Protegidas del Sureste Mexicano (Figura 1).

\section{Los municipios de Catemaco y San Andréstuxtla y sus localidades de estudio}

La población de Catemaco es de 46,702 habitantes distribuidos en 275 localidades, mientras que San Andrés Tuxtlas posee 148,447 habitantes y 234 localidades (INEGI, 2006). En ambos municipios los suelos los suelos son ricos en nutrientes y por su alto riesgo de erosión, deben manejarse con mucha precaución cuando se utilizan en actividades como agricultura y ganadería (INEGI, 2007). Ambos municipios y las localidades de estudio se presentan en la Figura 2.

Los porcentajes de Población Económicamente Activa (PEA) que se emplea en cada sector económico se presentan para los dos municipios en el Cuadro 1, observándose que en San Andrés la principal actividad económica es la agropecuaria seguida del sector terciario, mientras en Catemaco el sector terciario turístico es el principal, seguido del sector primario.

En cuanto al sector primario, en San Andrés Tuxtla los principales productos agrícolas son maíz, mango, tabaco, caña de azúcar, frijol, sandía, plátano, naranja y chile verde, mientras en Catemaco los cultivos importan- 


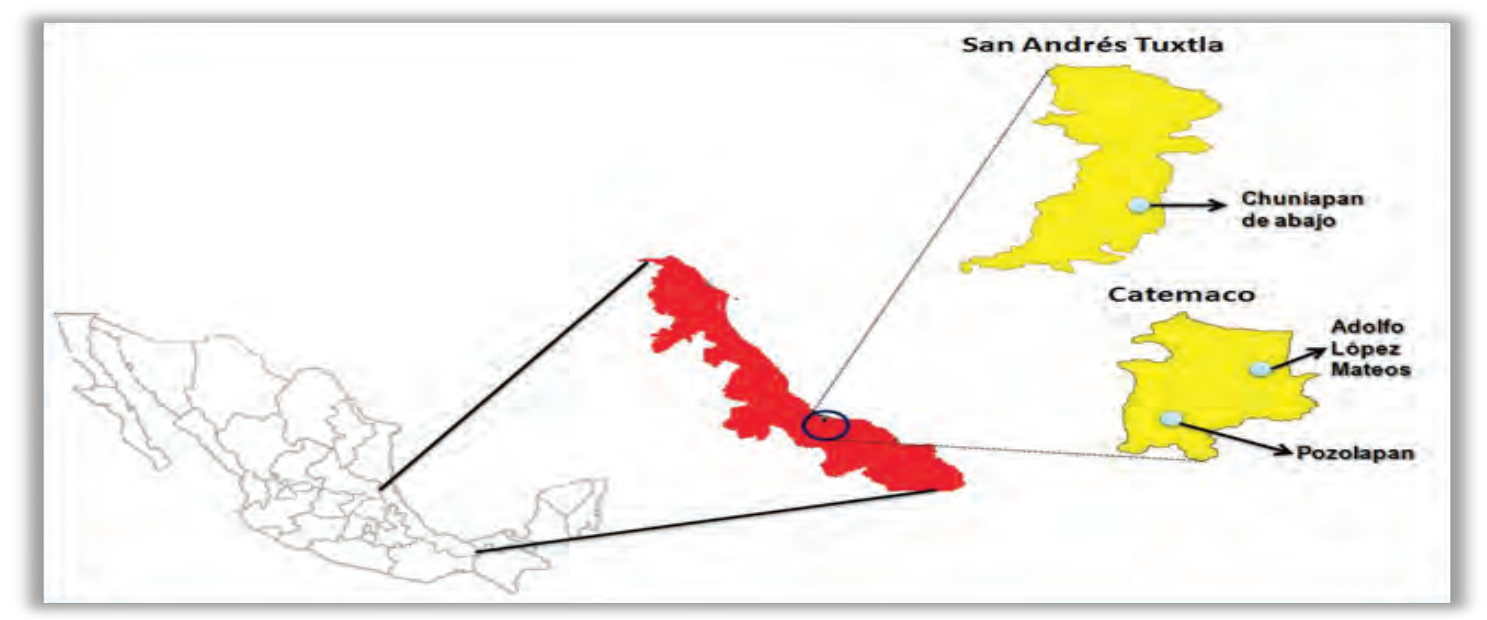

Figura 2. Localización de los municipios de Catemaco y San Andrés Tuxtla. Comunidades de estudio.

\begin{tabular}{|c|c|c|c|c|}
\hline \multirow{2}{*}{ Municipio } & \multicolumn{4}{|c|}{ SECTOR PRODUCTIVO (\%) } \\
\cline { 2 - 5 } & Primario & Secundario & Terciario & $\begin{array}{c}\text { No } \\
\text { especificado }\end{array}$ \\
\hline Catemaco & 40.2 & 14.8 & 43.4 & 1.6 \\
\hline San Andrés Tuxtla & 40.4 & 20.4 & 37.6 & 1.6 \\
\hline
\end{tabular}

Cuadro 1. Porcentaje de la PEA por sector productivo en Los Tuxtlas. 2000. Fuente: Elaboración propia según datos de INEGI, 2001.

tes son maíz, frijol, tabaco y chile (INEGI, 2006). En ambos municipios también es relevante la producción de ganado bovino, la pesca, la apicultura y la explotación forestal de maderas tropicales finas como cedro y caoba (CEDEMUN, 2000; Municipio de Catemaco 2007; Municipio de San Andrés Tuxtla, 2007).

En el sector de servicios turísticos, en Catemaco para el año 2006 se reportan 30 establecimientos de hospedaje y 38 restaurantes (INEGI, 2007). El municipio cuenta con una gran riqueza natural y una larga tradición en turismo de naturaleza; entre los centros turísticos relevantes se encuentran: Laguna de Catemaco, Reserva Ecológica Nanciyaga, Laguna de Sontecomapan, Playas como Hicacal, Playa Escondida y Monte Pío (Municipio de Catemaco, 2007). Mientras en San Andrés Tuxtla en

2006 existían 39 establecimientos de hospedaje, 19 restaurantes y dos agencias de viajes. Entre los principales atractivos turísticos del municipio se encuentran: Cascada Salto de Eyipantla, Laguna Encantada, Laguna Escondida y Playa Roca Partida, entre otros (INEGI, 2007). Estas comunidades y otras son comparables gracias al índice de pobreza. La Organización de las Naciones Unidas tiene un índice de pobreza integrado por Pobreza Alimentaria, Pobreza de Capacidades y Pobreza de Patrimonio. La pobreza alimentaria se refiere a la falta de capacidad para adquirir y consumir alimentos; la de capacidades a la pobreza de las personas que no pueden adquirir productos como vestimenta y accesorios básicos. La pobreza de patrimonio se refiere a la falta de recursos para adquirir una vivienda propia (PNUD, 2005). En cuanto a las condiciones sociales de los municipios estudiados, el Índice de Rezago Social para ambos es medio, mientras para el estado de Veracruz es alto. Este índice se refiere a las condiciones de vida de la población y se estima calculando el porcentaje de población que tiene acceso a la educación, servicios de salud, agua entubada, drenaje, calles pavimentadas, así como la proporción de casas con un solo cuarto. Sin embargo, si se analiza el Índice de Pobreza (Cuadro 2) se observa que los valores para los tres tipos de pobreza en ambos municipios son mayores que para el estado de Veracruz.

Respecto a la migración, en el Cuadro 3 se observa que San Andrés tiene más migrantes en otros estados de la república mexicana y en Estados Unidos que Catemaco.

\begin{tabular}{|c|c|c|c|}
\hline Lugar & Alimentaria & $\begin{array}{c}\text { De } \\
\text { capacidades }\end{array}$ & $\begin{array}{c}\text { De } \\
\text { patrimonio }\end{array}$ \\
\hline Veracruz & 28 & 36.3 & 59.3 \\
\hline Catemaco & 43.8 & 55.5 & 79 \\
\hline San Andrés Tuxtla & 48.9 & 59.3 & 80.4 \\
\hline
\end{tabular}

Cuadro 2. Índice de Pobreza. 2005. Fuente: CONAPO, 2006. 


\begin{tabular}{|c|c|c|c|c|c|c|c|c|c|c|c|}
\hline \multirow[t]{2}{*}{ Municipio } & \multirow{2}{*}{$\begin{array}{c}\text { Población } \\
\text { Total } \\
\text { Total }\end{array}$} & \multicolumn{2}{|c|}{ No migrante } & \multicolumn{2}{|c|}{$\begin{array}{c}\text { Migrante } \\
\text { estatal }\end{array}$} & \multicolumn{2}{|c|}{$\begin{array}{c}\text { Migrante } \\
\text { en E.U }\end{array}$} & \multicolumn{2}{|c|}{$\begin{array}{c}\text { Migrante en } \\
\text { otro país }\end{array}$} & \multicolumn{2}{|c|}{$\begin{array}{c}\text { No } \\
\text { especificado }\end{array}$} \\
\hline & & Total & $\%$ & Total & $\%$ & Total & $\%$ & Total & $\%$ & Total & $\%$ \\
\hline Catemaco & 41645 & 41,005 & 98.46 & 561 & 1.35 & 33 & 0.08 & 9 & 0.02 & 37 & 0.09 \\
\hline San Andrés Tuxtla & 133312 & 131,246 & 98.45 & 1,710 & 1.28 & 102 & 0.08 & 14 & 0.01 & 240 & 0.18 \\
\hline
\end{tabular}

Cuadro 3. Tipos de migrantes en Los Tuxtlas. 2000. Fuente: COESPO, 2002.

\section{La migración en Catemaco: Pozolapan y López Mateos}

El Conteo de Población 2005 (INEGI, 2006) reporta que Pozolapan tiene una población total de 702 habitantes. En lo relativo a migración, para el año 2000 residían en la localidad 606 personas de 5 años y más, sólo 4 personas residían en otra entidad federativa y ninguna radicaba en Estados Unidos (INEGI, 2006). Estos datos, no coinciden con los migrantes registrados en el trabajo de campo, debido a que en el Conteo de Población 2005, para captar la migración se pregunta sobre el lugar de residencia en el quinquenio anterior (2000), cuando la migración internacional recién se intensifica.

Pozolapan es una comunidad rural, por tanto la principal actividad económica es la agricultura minifundista de subsistencia. Los principales cultivos son maíz, cacahuate, café, frijol y pasto para ganado. Los productos agrícolas se destinan mayormente al autoconsumo. Algunas familias combinan la agricultura con crianza de ganado vacuno a pequeña escala, principalmente en pastoreo extensivo y con un manejo tecnológico mínimo.

En Pozolapan se aplicó una encuesta a 25 familias con migrantes nacionales y/o internacionales activos o con experiencia previa, registrándose datos para un total de 138 personas que son miembros de las familias entrevistadas, de éstos, 99 (71.7\%) permanecen en la comunidad y 39 (28.3\%) son migrantes que laboran de manera permanente fuera de la comunidad. Los datos de los migrantes se obtuvieron de manera indirecta, pues en la mayoría de los casos las personas que contestaron la encuesta fueron familiares de los ausentes. Es importante mencionar que de las 25 familias encuestadas 24 tienen migrantes y una actualmente no tiene ningún miembro trabajando fuera pero ha tenido en el pasado miembros migrantes.

El promedio de migrantes por familia es de 1.6. La edad promedio de los migrantes es de 29 años. En el rango de edad 16-30 años se encuentra el 59\% de los migrantes y el restante 41\% en el rango 31-50 años, es decir, son población en edad productiva que emigra como mano de obra en busca de mejores oportunidades de empleo.
El promedio de escolaridad de los migrantes es de 8.1 años (algún grado de secundaria), el cual es mayor al promedio de escolaridad de los que radican en la comunidad (5.3 años, que equivale a primaria incompleta). En lo relativo a la última ocupación de los migrantes en el lugar de destino (Figura 3), se aprecia que la mayoría de éstos (94.9\%) se desempeña en actividades del sector secundario y terciario contra un pequeño número empleado en el sector agrícola (5\%).

\section{MIGRANTES DE POZOLAPAN POR ÚLTIMO LUGAR DE TRABAJO EN LUGAR DE DESTINO}

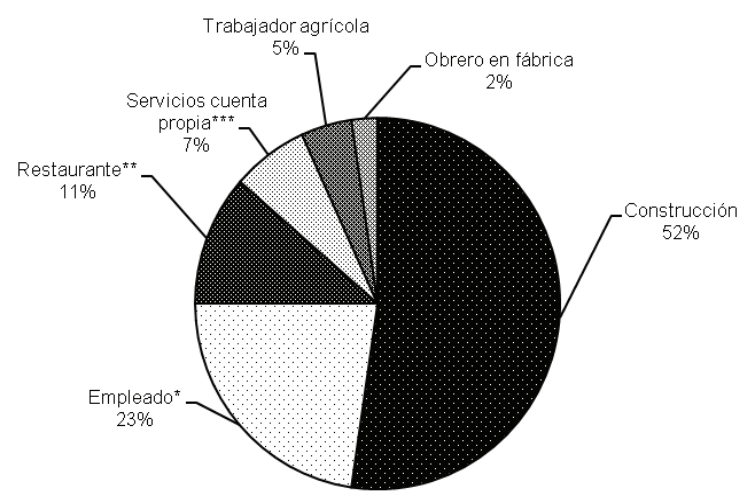

Figura 3. Migrantes de Pozolapan según última ocupación en lugar de destino. 2008.

La Figura 4 muestra el predominio de la migración internacional, siendo Carolina del Norte, el lugar de destino más frecuente. En el caso de la migración nacional, los destinos más registrados son el estado de Oaxaca y la ciudad de México D.F. De los 39 migrantes, 97.4\% (38) enviaba remesas a sus familiares en el pueblo y sólo un migrante $(2.6 \%)$ no. De las 25 familias encuestadas, todas han recibido en algún momento remesas que provienen mayormente de Estados Unidos. Aunque el envío de remesas tiene una periodicidad variable, lo más común es que se reciban cada mes.

En cuanto a lugar de destino, de un total de $39 \mathrm{mi}-$ grantes, $6(15.4 \%)$ residen en el país y 33 (84.6\%) en Estados Unidos. Las remesas enviadas por los migrantes 


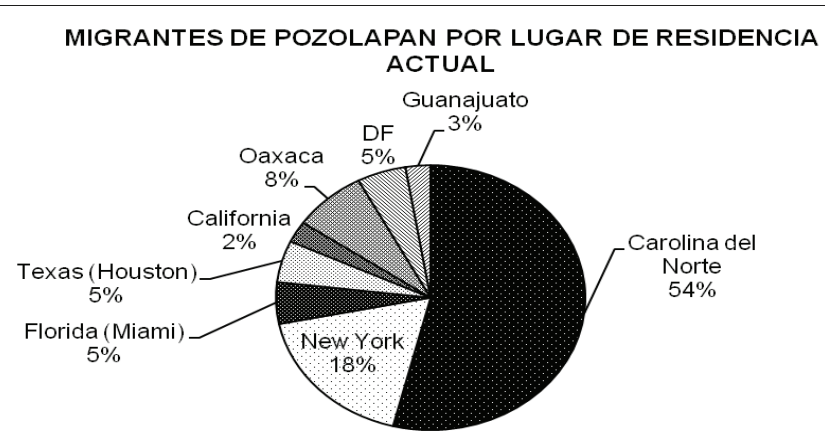

Figura 4. Migrantes de Pozolapan según lugar de residencia actual. 2008.

tienen más de un uso (Figura 5): el destino prioritario es el gasto familiar básico (alimentación, ropa, gastos médicos y escolares), seguido de mejora de vivienda. La inversión productiva en actividades agropecuarias u otro tipo de negocios es mínima. Ninguna familia mencionó invertir las remesas en alguna actividad relacionada con el ecoturismo.

Si se analiza el año de la primera salida de las personas con experiencia migratoria, se observa que el flujo migratorio se incrementa a partir del año 2000, ya que en el periodo 2000-2008, se registra la primera salida del $79.5 \%$ de este grupo. En cuanto al motivo de la primera migración, la mayoría de los migrantes (93.2\%) salieron por razones económicas (búsqueda de trabajo, mejora de ingreso, pago de deudas, pobreza, crisis del campo y ahorro para mejorar la vivienda), el resto (6.6\%) mencionó como motivos la aventura o la influencia de otros migrantes. El 81.8\% de los miembros con experiencia migratoria se dedicaba a las actividades agrícolas antes de salir de la comunidad.

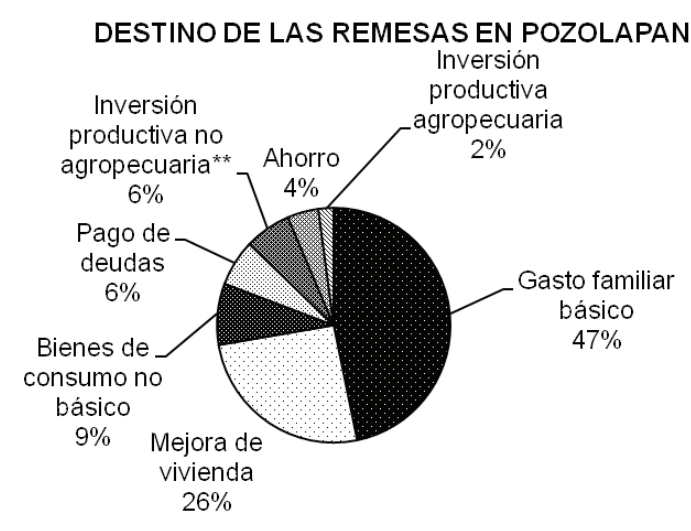

Figura 5. Destino de las remesas en Pozolapan. 2008.
En cuanto a los efectos que se perciben en la comunidad a raíz del aumento de la migración (Cuadro 4), existe muy poca diferencia entre los efectos positivos y negativos visualizados (alrededor de la mitad de las respuestas se inclinan por uno u otro): el efecto positivo más mencionado es la mejora de vivienda y el negativo más recurrente es la desintegración familiar.

Para la comunidad de López Mateos, el Conteo de Población 2005 reporta una población total de 114 habitantes. En el aspecto migratorio, se reporta que para el año 2000 ninguna persona residía en otra entidad federativa o en Estados Unidos (INEGI, 2006).

López Mateos es una comunidad rural que se encuentra en los límites de la zona de amortiguamiento y la zona núcleo de la Reserva de la Biosfera de Los Tuxtlas, por ello existe una gran diversidad de fauna y vegetación que han hecho posible consolidar la actividad ecoturística. La comunidad es parte de la Red de Ecoturismo Comunitario de los Tuxtlas (RECT) y posee una trayectoria de 12 años trabajando en el sec-

\begin{tabular}{|c|c|c|}
\hline TIPO DE EFECTO & FRECUENCIA* & $\%$ \\
\hline \multicolumn{3}{|l|}{ POSITIVOS } \\
\hline Mejora de la vivienda & 9 & 27.2 \\
\hline Compra de vehículo & 2 & 6.1 \\
\hline Mejora del nivel de vida familiar & 2 & 6.1 \\
\hline Compra de solares & 2 & 6.1 \\
\hline Aumento y mejora de ganadería & 2 & 6.1 \\
\hline Mayor inversión en actividades agropecuarias & 1 & 2.9 \\
\hline Subtotal & 18 & 54.5 \\
\hline \multicolumn{3}{|l|}{ NEGATIVOS } \\
\hline Desintegración familiar & 8 & 24.3 \\
\hline Escasez de mano de obra & 3 & 9.1 \\
\hline Pérdida de cultura rural & 2 & 6.1 \\
\hline Aumento de delincuencia & 1 & 3.0 \\
\hline Intensificación del trabajo para los que se quedan & 1 & 3.0 \\
\hline Subtotal & 15 & 45.5 \\
\hline TOTAL & 33 & 100 \\
\hline
\end{tabular}

Cuadro 4. Efectos de la migración en la comunidad de Pozolapan. 2008. * Frecuencia de respuesta: indicaron más de un cambio.

tor. La agricultura es otra actividad económica importante, basada en los cultivos de maíz y frijol, destinada mayormente a autoconsumo, atendida básicamente con mano de obra familiar y con un nivel tecnológico bajo (RECT, 2009).

A diferencia de otras comunidades, donde la migración internacional se ha incrementado progresivamente, en López Mateos parece haberse frenado, dado que 
al momento de realizar el trabajo de campo (2008) sólo pudieron identificarse cuatro familias con 7 migrantes internacionales, que estaban en Carolina del Norte, trabajando en el sector servicios. Un efecto indirecto que han tenido las remesas es el impulso del ecoturismo, dado que parte de las remesas se han invertido en esta actividad, impulsada sobre todo por la Red de Ecoturismo de los Tuxtlas (RECT).

"La actividad ecoturística ha logrado frenar la migración, (...). Iniciamos el proyecto con el apoyo de Luisa Paré y luego con la Reserva de la Biosfera y con la Red de Ecoturismo de los Tuxtlas (RECT) para que los compañeros no emigraran de la comunidad. De taladores de la selva a empresarios de ecoturismo, no es fácil pero le estamos echando ganas" (López Mateos, 28 de marzo de 2009).

La expectativa de muchos pobladores que alguna vez fueron migrantes es seguir mejorando y creciendo en el sector eco turístico, pero sin dejar de lado otras actividades económicas presentes en la comunidad, bien en el sector agropecuario, bien en el sector artesanal (madera y semillas).

Emigrar ha permitido a muchos de los habitantes comparar su lugar de origen con otras regiones y darse cuenta de la importancia de la Reserva de la Biosfera y los servicios ambientales que proporciona. De esta forma, la comunidad de López Mateos parece haber encontrado en el ecoturismo una actividad alternativa a la migración hacia Estados Unidos y a la vez (aunque resulte paradójico), la migración a través de las remesas, constituye una fuente de financiamiento para impulsar la actividad eco turística.

\section{La migración en San Andrés Tuxtla: Chuniapan de Abajo.}

El Conteo de Población 2005 reporta que Chuniapan de Abajo cuenta con una población total de 1040 habitantes. En aspectos de migración, para el año 2000 residían en el poblado 915 personas de 5 años y más, sólo 2 personas (para el mismo año y rango de edad) vivían en otra entidad federativa y ninguna radicaba en Estados Unidos (INEGI, 2006). Chuniapan de Abajo es una comunidad rural, dedicada esencialmente a la producción agrícola minifundista, que utiliza mayormente mano de obra familiar, orientada al autoconsumo y escasamente tecnificada.

En Chuniapan de Abajo se aplicó una encuesta a 27 familias con migrantes nacionales y/o internacionales activos o con experiencia previa, registrándose datos para un total de 146 personas que son miembros de las familias entrevistadas, de éstos, 110 (75.3\%) permane- cen en la comunidad y 36 (24.7\%) son migrantes que laboran de manera permanente fuera del pueblo. De las 27 familias encuestadas, 23 tienen migrantes activos y 4 actualmente no tienen ningún miembro trabajando fuera pero han tenido en el pasado miembros migrantes.

El promedio de migrantes por familia es de 1.6. La edad promedio de los migrantes es de 28 años y su promedio de escolaridad es de 6.8 años (primer grado de secundaria), el cual es mayor al promedio de escolaridad de los que radican en la comunidad (4.3 años que corresponde a primaria incompleta).

En lo relativo a la última ocupación de los migrantes en el lugar de destino (Figura 6), se aprecia que más de la mitad se desempeña como empleado, el resto en restaurantes $\mathrm{u}$ otras actividades del sector secundario y de servicios; no se registró ningún migrante laborando en

\section{MIGRANTES DE CHUNIAPAN DE ABAJO POR ÚLTIMO TRABAJO EN LUGAR DESTINO}

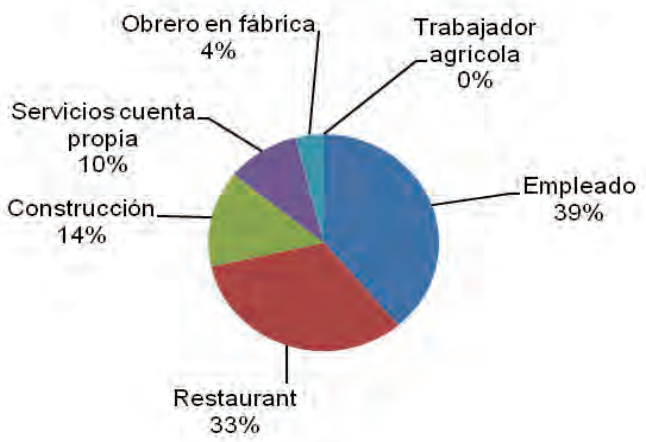

Figura 6. Migrantes de Chuniapan de Abajo según última ocupación en lugar de destino. 2008.

el sector agrícola.

En lo relativo a lugar de destino, de un total de 36 migrantes, sólo uno (2.8\%) reside en el país y 35 (97.2\%) en Estados Unidos. Los datos de la Figura 7 muestran el predominio de la migración internacional, siendo la

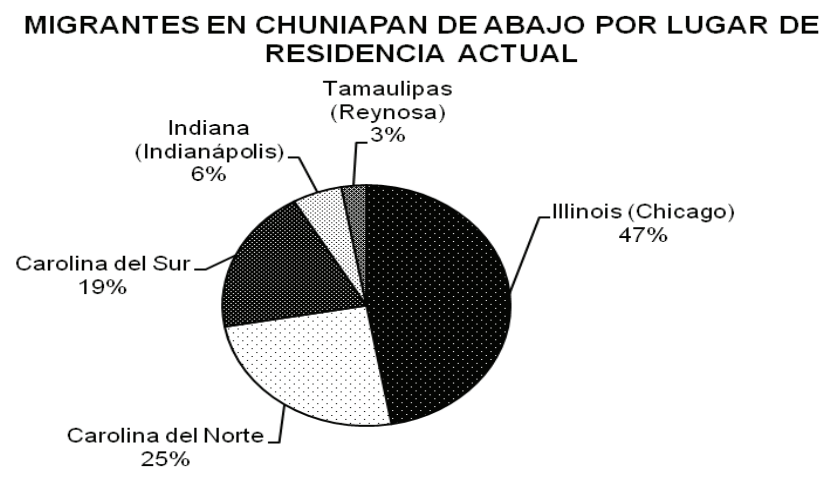

Figura 7. Migrantes de Chuniapan de Abajo según lugar de residencia actual. 2008. 
ciudad de Chicago, Illinois, el destino más frecuente.

De los 36 migrantes activos, 94.4\% (34) envían remesas a sus familiares en el pueblo y sólo dos migrantes no. Así, de las 27 familias encuestadas, todas han recibido en algún momento remesas y actualmente $74 \%$ (20 familias) las reciben aproximadamente cada mes, de los migrantes que laboran en Estados Unidos. Las remesas se destinan a más de un uso (Figura 8), el destino prioritario es el gasto familiar básico (alimentación, ropa, gastos médicos y escolares), seguido de mejora de

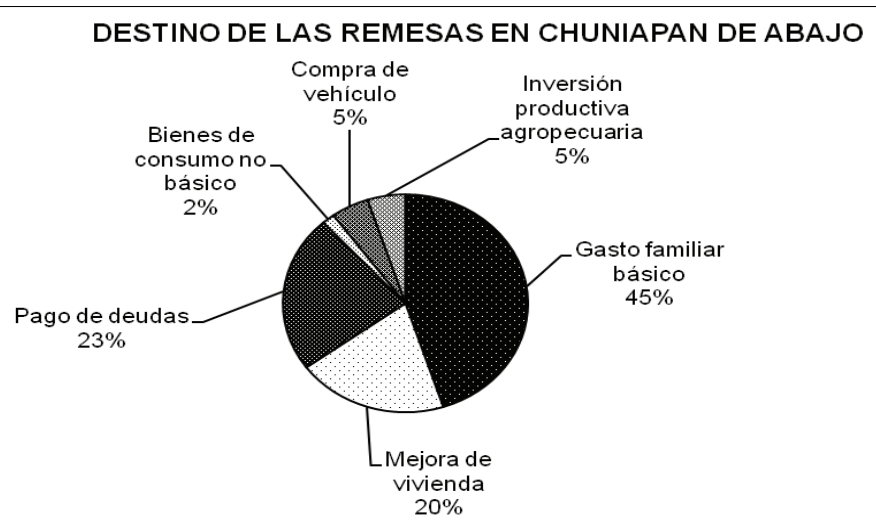

Figura 8. Destino de las remesas en Chuniapan de Abajo. 2008.

\begin{tabular}{|c|c|c|}
\hline TIPO DE EFECTO & Frecuencia* & $\%$ \\
\hline \multicolumn{3}{|l|}{ POSITIVOS } \\
\hline Mejora vivienda & 5 & 9.7 \\
\hline Aumento y mejora de ganadería & 4 & 7.7 \\
\hline Apertura de pequeños comercios & 2 & 3.8 \\
\hline Compra de solares & 1 & 1.9 \\
\hline Inversión en actividades agropecuarias & 1 & 1.9 \\
\hline Subtotal & 13 & 25 \\
\hline \multicolumn{3}{|l|}{ NEGATIVOS } \\
\hline Disminución o abandono de actividad agrícola & 10 & 19.3 \\
\hline Desintegración familiar & 10 & 19.3 \\
\hline Aumento de alcoholismo & 6 & 11.5 \\
\hline Escasez de mano de obra & 4 & 7.7 \\
\hline Incremento de enfermedades urbanas (gastritis, diabetes) & 3 & 5.8 \\
\hline Intensificación del trabajo para los que se quedan & 2 & 3.8 \\
\hline Envejecimiento de la población residente & 2 & 3.8 \\
\hline Pérdida de cultura rural & 1 & 1.9 \\
\hline Endeudamiento de migrantes que fracasan & 1 & 1.9 \\
\hline Subtotal & 39 & 75 \\
\hline TOTAL & 52 & 100 \\
\hline
\end{tabular}

Cuadro 5. Efectos de la migración en la comunidad de Chuniapan de Abajo. 2008. * Frecuencia de respuesta: indicaron más de un cambio. vivienda y pago de deudas. La inversión productiva en actividades agropecuarias es mínima y no se registró ninguna inversión destinada a otro sector económico (servicios, turismo, comercio, etc.).

La migración se incrementa a partir del año 2000, ya que entre este y el 2008 en que se levantó la encuesta, se registra la primera salida del $89.8 \%$ de los encuestados con experiencia migratoria. Las 49 personas con experiencia migratoria (100\%) expresaron que el motivo de la primera migración involucró razones económicas que incluyen búsqueda de trabajo, mejora de ingreso, pago de deudas, pobreza y crisis del campo. En cuanto a la ocupación de los miembros con experiencia migratoria antes de salir de la comunidad, $91.9 \%$ se dedicaban a las actividades agrícolas.

En cuanto a los efectos que se observan en la comunidad a raíz del aumento de la migración (Cuadro 5), se visualizan más los cambios negativos, siendo los más mencionados la disminución o abandono de la actividad agrícola y la desintegración familiar. Mientras que los positivos más recurrentes son la mejora de vivienda y la mejora de la ganadería.

\section{Ecoturismo en la región de los Tuxtlas}

El turismo de naturaleza en la región de Los Tuxtlas se practica en 13 localidades, en las que existen 14 empresas eco turísticas. Éstas cuentan con servicios básicos de hospedaje y alimentación y, sin embargo, adolecen de servicios complementarios que enriquezcan la experiencia turística. A ello se agrega una débil infraestructura de vías de comunicación y la falta de inversión pública para mejorar las rutas de acceso, lo cual dificulta llegar a las comunidades con oferta ecoturística. En el mismo sentido, la infraestructura (agua potable, energía eléctrica, medios de comunicación, tratamiento de desechos sólidos y aguas residuales) es regular o mala en las localidades que tienen negocios ecoturísticos, es decir, las necesidades de infraestructura de soporte constituyen una seria limitante para los desarrollos eco turísticos regionales. A pesar de las deficiencias en materia de servicios complementarios, vías de comunicación e infraestructura, las comunidades de la región de Los Tuxtlas han logrado importantes avances en el sector eco turístico.

Ecoturismo comunitario en Adolfo López Mateos o "Selva el Marinero"

La comunidad de Adolfo López Mateos pertenece al municipio de Catemaco y se encuentra en los límites de la zona de amortiguamiento y la zona núcleo de La Reserva de la Biosfera de Los Tuxtlas, por ello se aprecia fauna y vegetación diversa. 
La génesis del ecoturismo en los Tuxtlas se produce en López Mateos en la década de 1990, a partir de ello se han multiplicado las empresas eco turísticas privadas y comunitarias en distintas localidades, promovidas por la acción de investigadores que han trabajado en la zona y la declaración de los Tuxtlas como parte de la Reserva de la Biosfera en 1998. Un paso importante en este proceso se da en 1993, cuando la comunidad de López Mateos en asamblea ejidal decide la restricción voluntaria del uso de sus recursos naturales para la conservación de 100 ha de terreno de selva virgen. Será hasta 1997 cuando se implemente un proyecto de ecoturismo en la Selva del Marinero. Así, desde 1998 se ha trabajado en la consolidación de la empresa ecoturística, la cual se constituyó legalmente en una Sociedad de Solidaridad Social, denominada "Cielo, Tierra y Selva, Reserva del Marinero", que en la actualidad cuenta con 24 socios ejidatarios.

López Mateos ha sido pionera en construir el primer centro ecoturístico comunitario dentro de la Reserva de la Biosfera de los Tuxtlas. Los servicios ofrecidos por López Mateos como parte de la Red de Ecoturismo Comunitario de los Tuxtlas son: hospedaje en cabañas; servicio de comedor en las casas de los campesinos; recorridos por senderos interpretativos (ríos, lagos y lagunas); observación de aves; visita a los jabalíes; elaboración de artesanías de madera de cocuite, coco y cuerno de res (para fabricar artesanías se utiliza sólo madera decomisada o derribada por los huracanes). Para 2008 la empresa comunitaria eco turística de López Mateos empleaba 35 personas de manera permanente. Es importante mencionar que algunas de las familias que rcibían remesas de Estados Unidos las invirtieron en el ecoturismo.

\section{Ecoturismo privado en Pozolapan}

La comunidad de Pozolapan está situada a la orilla del lago de Catemaco, pero no es parte de la Reserva de la Biosfera de los Tuxtlas, y posee una corta trayectoria en la actividad ecoturística. Existen dos empresas privadas: El Teterete y Bahía Escondida, impulsadas por empresarios con estudios universitarios, que llegan inmigran a la comunidad en 1978 y 2001, provenientes del Distrito Federal y Suiza, respectivamente. Estas empresas ofrecen servicios ecoturísticos dentro de la comunidad de Pozolapan, para la Reserva de la Biosfera y con otras empresas ecoturísticas privadas y comunitarias que operan en localidades de la región. A continuación se caracterizan ambas empresas.

\section{Pozolapan: Centro Ecoturístico "El Teterete"}

Las ideas de ecoturismo en la comunidad de Pozolapan surgen a raíz de la llegada de Roberto Barrón en 1978, quien junto con su esposa trabaja en aspectos de planificación familiar. En esta época, trabajaron con jóvenes de la comunidad en programas de salud reproductiva y en el establecimiento de criaderos de tegogolos (caracoles de lago) y mojarras. Más adelante surge la idea del ecoturismo:

“(...) vimos que la planificación familiar se había superado y la infraestructura creada había que mantenerla y se tenía que autofinanciar (...). Fue cuando dijimos vamos a abrirlo como negocio de alojamiento y restaurantito". (Pozolapan, $12 \mathrm{de}$ julio 2008)

Respecto a la generación de empleo, en "El Teterete" la gente contratada es mínima por el carácter familiar del negocio, y por ser el ecoturismo una actividad estacional que funciona con baja capacidad de carga de visitantes para no deteriorar los recursos naturales. El empleo generado varía dependiendo de la afluencia de turistas en la temporada vacacional y fines de semana, pero se contratan regularmente de una a cinco personas de planta. A éstos se les capacita con la idea de conservar el recurso natural con actividades de bajo impacto: "Trabajadores fijos son la cocinera, el que limpia y el que me ayuda al mantenimiento, ellos son de Pozolapan. Por el momento sólo trabajamos los fines de semana (sábado y domingo) en el restaurante. No trabajamos entre semana, por otras actividades laborales, a veces empleamos a personas de otras comunidades cercanas; a todos se les capacita en ecoturismo." (Pozolapan, 12 de julio de 2008)

A pesar de esta limitante, El Teterete es un establecimiento ecoturístico privado, que contribuye tanto a la conservación del entorno natural como al desarrollo económico local, dado que contrata trabajadores de la comunidad, constituyendo una fuente de empleo e ingreso para algunas familias de Pozolapan. En cuanto a los servicios ofrecidos, el lugar cuenta con: renta de cabañas; renta de salón para eventos; alberca; herpetario; servicio de comedor los fines de semana, vacaciones y días festivos. Además, ofrece recorridos por senderos en la comunidad de Pozolapan y en lanchas tipo velero por el lago de Catemaco, así como visitas a los sitios "La Joya", "Dos Amates", al "brujo" y a conocer el trabajo de los pescadores. 


\section{Pozolapan: Centro Ecoturístico "Bahía Escondi- da"}

Este desarrollo ecoturístico privado es fundado por un suizo, que después de recorrer México durante dos décadas decide asentarse en Pozolapan:

"Yo soy suizo de origen y llegué a México en los años 80. Es un país maravilloso, rico en cultura, con un ritmo de vida tranquilo. De los países que conozco en Europa y Latinoamérica, he preferido este país. He viajado durante 15 años por toda la República mexicana, vendiendo artesanía, platería y piel. Conozco bien todo el país y mi hija nació en Chiapas. Me quedé en Los Tuxtlas porque me trataron muy bien, la gente es amable y educada. Me ha gustado viajar a Catemaco. Después de unos años compramos en Pozolapan en el año 2001, abrimos el negocio a final de 2002, empezamos con la página web que es la que nos jala muchísima gente desde el año 2004. Iniciamos yo y mi hija” (Pozolapan, 26 de marzo de 2009)

Este establecimiento de ecoturismo se ha orientado hacia el turismo "mochilero" nacional y extranjero, sobre todo estudiantes universitarios, académicos e investigadores preocupados por la conservación de los recursos naturales. Si bien "Bahía Escondida" está fuera de la Reserva de la Biosfera de Los Tuxtlas, también se benefició de la declaración de la región como parte de esta Área Natural Protegida, ya que su oferta ecoturística se basa en ofrecer al visitante dos aspectos esenciales: naturaleza y buen trato.

En la actualidad, Bahía Escondida ofrece los siguientes servicios: 1) alojamiento diversificado según el tipo de visitante (cabaña, dormitorios y camping); 2) comedor y cocina donde también los visitantes pueden preparar sus comidas, comprando alimentos en los negocios del pueblo; 3) venta de artesanías; 4) aunque la empresa no cuenta con recorridos establecidos, las conexiones con otros negocios ecoturísticos permiten que los visitantes realicen recorridos por la selva, senderos interpretativos, visita a cuevas, cascadas, lagos, lagunas, ríos, observación de la naturaleza, observación de aves, recorridos en lancha, visita de sitios arqueológicos, cabalgatas, rappel y descenso en ríos.

En cuanto a generación de empleo, dado que es una empresa privada familiar, el empresario y su hija realizan la mayor parte de las actividades; sólo es necesario contratar empleados locales en época de mayor afluencia turística. Sin embargo, es evidente el afán empresarial de que exista empleo local y derrama económica en Pozolapan y en la región, así como que el impacto del visitante en el medioambiente sea reducido o nulo

\section{Discusión de los resultados}

En el presente apartado se discuten los resultados respecto a la migración y sus efectos, las características de la actividad ecoturística y la relación entre migración y ecoturismo en las comunidades estudiadas.

\section{(1) La migración y sus efectos en la región de los Tuxtlas}

En la región de estudio las migraciones no son movimientos de individuos, sino de fuerza de trabajo que es desplazada de algunas áreas poco desarrolladas (en este caso el medio rural) a otras regiones con mayor desarrollo económico (Estados Unidos), con el objetivo de lograr su reproducción biológica y social. Además, aunque el origen de la migración en Los Tuxtlas se relaciona principalmente con motivos económicos, su aumento y continuidad se debe a la creación de redes sociales, es decir, los migrantes van conformando en las sociedades receptoras, un conjunto de lazos con amistades y parientes que permanecen en las comunidades de origen; esto hace posible la perpetuación del flujo migratorio, dado que estas redes disminuyen el riesgo y costo para los nuevos migrantes.

La migración en las comunidades de estudio no es un fenómeno nuevo, sin embargo, a partir de finales de la década de los noventa y principios del presente siglo, se observa un crecimiento y predominio de la migración hacia Estados Unidos. De ahí que los datos estadísticos del Censo de Población del 2000 (INEGI, 2001) y del Conteo de Población 2005 (INEGI, 2006) no reflejen la realidad migratoria internacional captada en el trabajo de campo en los Tuxtlas, ya que en ambos estudios sólo se registran datos para los residentes permanentes del domicilio a la fecha de la encuesta (no se capta a los ausentes).

Los porcentajes de migrantes internacionales captados en las comunidades donde se aplicó la encuesta son importantes, ya que de los miembros de las familias incluidas en el estudio, los migrantes representaban 28.3\% en Pozolapan y 24.7\% en Chuniapan de Abajo. Mientras que a nivel nacional se reporta que para 2008, $11 \%$ del total de la población en México son migrantes que radican en Estados Unidos (Leite et al., 2009).

Los migrantes activos internacionales son población mayoritariamente masculina en edad productiva, con mayor promedio de escolaridad que los habitantes que residen en la comunidad, en su lugar de origen se dedican principalmente a la agricultura, pero en los lugares de destino (generalmente urbanos), trabajan en empleos poco calificados del sector servicios. Mediante las redes sociales, cada comunidad va consolidando lugares 
de destino específicos, ya que los migrantes no son individuos aislados, sino miembros de hogares, de grupos de parentesco y comunidades. En el caso de Chuniapan de Abajo, las redes migratorias internacionales se han consolidado principalmente hacia Illinois (Chicago), Carolina del Norte y Carolina del Sur. Mientras en Pozolapan esencialmente hacia Carolina del Norte y New York.

La comunidad de López Mateos es un caso especial, ya que aunque la migración inicia a finales de la década de los noventa hacia Carolina del Norte, constituyéndose igualmente una red social que incrementa paulatinamente el flujo de salida poblacional, durante el trabajo de campo de la presente investigación, sólo se identificaron 4 familias con 7 migrantes internacionales que estaban en Carolina del Norte, trabajando en el sector servicios. Lo cual indica una reversión del flujo migratorio internacional.

Las decisiones respecto a quién emigra, a dónde y con qué propósito, están determinadas tanto por factores del contexto socioeconómico mayor en que se insertan las comunidades (políticas agropecuarias neoliberales, crisis nacional del sector primario, endurecimiento de normatividad y control en la frontera norte), como por las condiciones al interior de las familias (determinaciones de género -emigran más los hombres-, relaciones de parentesco, jerarquía de poder y necesidades específicas dentro de los hogares).

Los efectos contradictorios de la migración observados en las comunidades de estudio, coinciden con lo reportado por diversos autores (Arango, 2003; Nava, 2009), quienes mencionan como efectos: 1) A nivel familiar, una sustancial mejora en calidad de vida y consumo, por lo que la migración se constituye a corto plazo en una estrategia de sobrevivencia familiar; 2) en la comunidad, un deterioro de la base económica y cultural que sustenta la producción agropecuaria (principal actividad económica) y propicia la desintegración de las familias rurales, por lo que paralelamente la migración constituye una problemática que puede desarticular el sector rural a largo plazo.

$\mathrm{Al}$ analizar los efectos de la migración en las comunidades estudiadas, aunque existen algunas coincidencias entre ellas, también se presentan diferencias importantes que se resumen a continuación:

Chuniapan de Abajo. Los hogares encuestados visualizan mayormente los efectos socioeconómicos negativos (75\% de las respuestas) que positivos (25\%). El efecto positivo de la migración se relaciona con las remesas que reciben los hogares y que se destinan en orden de importancia a: mejorar la vivienda, aumentar y mejorar el ganado, apertura de pequeños negocios, y en menor medida, comprar solares e invertir en actividades agropecuarias. Mientras que los efectos negativos más mencionados fueron: disminución o abandono de la actividad agrícola, desintegración familiar, aumento del alcoholismo, escasez de mano de obra y consecuentemente, intensificación de trabajo para los que se quedan en la comunidad; en menor medida también se mencionó el incremento de enfermedades urbanas (gastritis, diabetes), pérdida de la cultura rural, envejecimiento de la población residente y endeudamiento de migrantes que fracasan en su intento de cruzar la frontera.

Pozolapan. En esta comunidad se visualizan un poco más los efectos socioeconómicos positivos (54.5\% de las respuestas) e igualmente éstos se relacionan con las remesas que se invierten mayormente en la mejora de vivienda y en menor medida en incrementar el nivel de vida familiar, comprar vehículos o solares e inversión en actividades agropecuarias. Por el contrario, el 45.5\% de las respuestas se refieren a efectos negativos de la migración como: desintegración familiar, escasez de mano de obra e intensificación del trabajo para los miembros que se quedan en la comunidad, pérdida de la cultura rural y aumento de la delincuencia.

López Mateos. Los entrevistados coinciden en señalar que los efectos positivos de la migración se dan en el ámbito económico a través de la inversión de las remesas en "construcciones y mejora de la calidad de vida". Mientras que el efecto negativo que ha dejado en la comunidad la partida de los hombres a Estados Unidos, ha sido principalmente la desintegración familiar.

\section{(2) La actividad ec otunística en la región de los Tuxt- las}

Los municipios seleccionados tienen las características necesarias para desarrollar exitosamente actividades de ecoturismo: recursos naturales sobresalientes, accesibilidad (mejorable), calidad de los servicios, calidez humana y cercanía con los principales centros urbanos y destinos turísticos de interés regional (Bigné; Font y Andreu, 2000; Acerenza 2007). En la zona de estudio se identificaron tres empresas eco turísticas en el municipio de Catemaco:

Selva del Marinero: empresa ecoturística comunitaria del ejido López Mateos, fundada en 1997, organizada como Sociedad de Solidaridad Social conformada por 24 socios ejidatarios. Cuenta con infraestructura básica de hospedaje y alimentación, recorridos y diversas actividades ecoturísticas; está integrada a la Red de Ecoturismo Comunitario de los Tuxtlas, genera 35 empleos fijos para gente de la comunidad.

Centro Ecoturístico "El Teterete": empresa ecoturística privada localizada en Pozolapan, iniciada en 2003 por un profesionista que llega a trabajar a la comuni- 
dad, cuenta con infraestructura básica de hospedaje y alimentación, algunos recorridos y actividades ecoturísticas; dado que es un negocio de carácter familiar y que la actividad ecoturística es estacional y de baja capacidad de carga, el personal que se contrata es mínimo. El empleo generado varía dependiendo de la afluencia de turistas en la temporada vacacional y fines de semana, pero se contratan regularmente de una a cinco personas de la comunidad.

Bahía Escondida: empresa eco turística privada localizada en Pozolapan, iniciada en 2002 por un extranjero que se establece en la comunidad, cuenta con infraestructura básica de hospedaje y alimentación; no ofrece recorridos pero conecta a sus clientes con otros negocios ecoturísticos de la región que proporcionan este servicio. En cuanto a generación de empleo, dado que es una empresa privada familiar, el empresario y su familia realizan la mayor parte de las actividades; sólo es necesario contratar empleados locales en época de mayor afluencia turística (dos empleados). En todos los casos persiste la preocupación por la conservación de la naturaleza de las empresas ecoturísticas abordadas, no está sólo motivada porque les proporciona una fuente de empleo e ingreso, sino que implica un verdadero interés en el cuidado del ambiente.

A pesar de que las empresas estudiadas tienen poco tiempo operando, son de carácter familiar, comunitarias o privadas, y operan a pequeña escala por la misma naturaleza de la actividad ecoturística (estacional y de baja capacidad de carga), los testimonios coinciden en señalar que la actividad ecoturística genera empleo local directo e indirecto y derrama económica en la región, por los eslabonamientos económicos que produce la presencia del ecoturista.

Aunque el efecto del ecoturismo, tanto en la conservación de los recursos naturales como en el desarrollo económico local, es aún limitado, presenta potencialidad para incrementarse a mediano y largo plazo, siempre y cuando se atiendan las problemáticas medioambientales y socioeconómicas que actualmente enfrenta el sector ecoturístico de la región de Los Tuxtlas.

\section{(3) Migrac ión y actividad ec otunística en la región de Los Tuxtlas}

La relación entre migración y ecoturismo no es unidireccional, sino que ambos fenómenos se influyen mutuamente. Por un lado, las remesas han contribuido (aunque en pequeña escala) a promover la actividad ecoturística, lo cual es más evidente en la comunidad de López Mateos, donde existe una empresa ecoturística campesina de beneficio comunitario. Por otra parte, en las comunidades estudiadas la actividad ecoturística genera efectos económicos que pueden incidir en el mercado laboral y por tanto en la disposición de la población a emigrar.

De forma directa, los negocios ecoturísticos crean empleos que permiten a algunas familias tener ingresos complementarios gracias al ecoturismo. Esta situación es más evidente en el caso de López Mateos, donde un número importante de personas son socios o empleados de la empresa ecoturística. En el caso de los negocios privados de Pozolapan la creación de empleos directos resulta mínima dado que son empresas familiares que operan a pequeña escala y de forma estacional.

De forma indirecta los negocios ecoturísticos, comunitarios y privados, impactan positivamente la economía local y regional en los periodos de temporada alta de turismo, bien dando empleo temporal a los pobladores, bien haciendo consumo de los bienes y servicios de otros negocios turísticos y no turísticos.

Según testimonios de los entrevistados, un efecto indirecto que han tenido las remesas enviadas por migrantes es el impulso del ecoturismo, dado que parte de éstas se han invertido en dicha actividad. Asimismo, la experiencia de los migrantes fuera de la comunidad, ha permitido que tomen conciencia de la importancia de los recursos naturales que poseen. Esta situación es más palpable en López Mateos donde los migrantes que han retornado, encuentran en el sector ecoturístico un ámbito rentable para invertir, generar recursos para sostenimiento familiar, construir un plan de vida que los arraigue a la comunidad y conservar su entorno natural y cultural.

Así, en López Mateos donde existe una empresa comunitaria consolidada que lleva operando desde el año 1998 y genera empleos directos e indirectos para la población, la actividad ecoturística parece haber frenado el flujo migratorio, pues aunque dicha comunidad tuvo el patrón migratorio ascendente de otros poblados de la región, actualmente los migrantes internacionales activos son mínimos y la expectativa de muchos pobladores que alguna vez fueron migrantes es seguir mejorando y creciendo en el sector ecoturístico.

En Pozolapan donde existen dos pequeñas empresas ecoturísticas, de caracter privado y familiar, que tienen menos tiempo operando y sólo contratan un reducido número de trabajadores asalariados durante la temporada alta de afluencia de turistas, el $28.3 \%$ de la población encuestada son migrantes activos, por lo que puede inferirse que a pesar de los beneficios económicos indirectos de la actividad ecoturística, la operación a pequeña escala de dichas empresas no ha logrado impactar significativamente el mercado laboral local, como para observar efectos de reversión del flujo migratorio. Los 
efectos económicos reducidos de éstas empresas, también se relacionan con la alta estacionalidad o presencia de visitantes sólo en períodos fijos y cortos del año (Semana Santa, verano, Navidad y fines de semana) y la escasa afluencia durante el resto del tiempo.

Lo anterior se corrobora al revisar el caso de Chuniapan de Abajo, donde a pesar de que existe interés en el ecoturismo, no han cristalizado las iniciativas para desarrollar empresas ecoturísticas y $24.7 \%$ de la población encuestada son migrantes activos, dato menor que el registrado en Pozolapan (28.3\%). Además, desde la perspectiva de los pobladores de Chuniapan de Abajo, los efectos económicos positivos de la migración (relacionados con la captación de remesas), no han podido contrarrestar sus efectos negativos; y por diversas problemáticas, las remesas no han logrado canalizarse hacia un sector de actividad económicamente redituable como el ecoturismo.

\section{Conclusiones}

Las consideraciones anteriores parecen indicar que el ecoturismo puede ser una alternativa económica viable para los pobladores de comunidades rurales marginadas en áreas naturales protegidas que todavía cuentan con recursos naturales y culturales valiosos, mayormente cuando en la constitución de la empresa ecoturística se fomenta la participación activa de la mayoría de familias de la comunidad, es decir, se promueven empresas comunitarias donde los pobladores son socios y acceden a la mayor parte de los beneficios. A largo plazo, estas empresas comunitarias y privadas pueden generar el empleo e ingresos (directos e indirectos) suficientes para impactar positivamente el mercado laboral local y regional, proporcionando a los pobladores un ámbito de actividad económica rentable para invertir, fincar proyectos de vida que los arraiguen a la comunidad y disminuir la necesidad de emigrar por motivos económicos.

En cuanto a si la actividad ecoturística contribuye a frenar la migración en las comunidades marginadas, debido que proporciona empleos e ingresos a los pobladores, que los arraigan a la comunidad, esto se observa para el caso de López Mateos donde existe una empresa eco turística comunitaria que ha creado un número importante de empleos directos e indirectos y donde actualmente existe escasa migración.

En el caso de Pozolapan es poco evidente el freno a la migración. En esta localidad existen sólo dos pequeñas empresas privadas familiares creadas por migrantes que generan un reducido número de empleos.

\section{Agradecimientos}

Al Fondo Mixto CONACYT-Gobierno del Estado de Veracruz, por financiar la presente investigación a través del proyecto "Efectos de la migración en la actividad agrícola de cuatro regiones campesinas del estado de Veracruz" (clave 68277).

\section{Bibliografía}

Acerenza, M. A.

2007. Desarrollo sostenible y gestión del turismo. México: Editorial Trillas.

Arango, J.

2003. "La explicación teórica de las migraciones: luz y sombra”. Migración y desarrollo. 1: 4-22.

Bigne J. ; X. Font y L. Andreu.

2000. Análisis y estrategias de desarrollo de los destinos turísticos, Madrid: Ed. ESIC,.

CEATUX (Comunidad de Educadores Ambientales de los Tuxtlas).

2009. Estrategia de Educación Ambiental para la Ecoregión los Tuxtlas y Sierra Santa Martha. Ms. Catemaco, Xalapa: SENDAS A.C.

CEDEMUN (Centro Nacional de Desarrollo Municipal). 2000. Enciclopedia de los Municipios de México (versión electrónica). Veracruz Vol. 4. México: CEDEMUN.

CONANP (Comisión Nacional de Áreas Naturales Protegidas).

2006. Programa de Conservación y Manejo en la Reserva de la Biosfera de los Tuxtlas. 2006. CONANP, DG de Manejo para la Conservación y la Dirección Regional Centro y Golfo México. http://www.conanp. gob.mx/ México

CONAPO.

2006. Índices de intensidad migratoria México Estados Unidos. CONAPO: México. (Consultada el 9 de septiembre de 2008). http://www.conapo.gob.mx/publicaciones/intensidadmig/CUA_AB_IIM2000.xls

COESPO (Consejo Estatal de Población).

2002. La migración en el Estado de Veracruz 1930-2000. Xalapa, México: Mimeo.

Chávez L., A. M.; C. A. Rosas y P. E. Zamudio G.

2007. "Cambios en la migración del estado de Veracruz. Consecuencia y retos. Red Internacional de Migración y Desarrollo". Consultada 12 de noviembre de 2007. http//: www.migracionydesarrollo.org

García C, H.

2009. "Educación ambiental y desarrollo regional: enfoque participativo en la Eco región de los Tuxtlas, Veracruz". Conferencia en el marco del ciclo de conferencias sobre desarrollo regional y medioambiente, 
Xalapa: El Colegio de Veracruz, 26/02/2009..

González A., J.A.

2008. "Reserva de la Biosfera Los Tuxtlas: Orgullo de Veracruz, patrimonio de la Nación". Conferencia en el marco del ciclo de conferencias sobre desarrollo regional y medioambiente, Xalapa: El Colegio de Veracruz, 26/06/2008.

Guevara S., S.; Laborde D., J. \& Sánchez-Ríos, G.

2006. Los Tuxtlas. El paisaje de la Sierra. México: Instituto de Ecología,

Hernández, R., C. Fernández y P. Baptista.

2006. Metodología de la Investigación. México: Mc Graw Hill.

INEGI 2001. XII Censo General de Población y Vivienda

2000. Síntesis de Resultados. Veracruz-LLave. INEGI ((Instituto Nacional de Estadística, Geografía e Informática). México.

2004. Cuaderno Estadístico Municipal. San Andrés Tuxtla. INEGI. México. (Versión electrónica)

2006. II Conteo de Población y Vivienda 2005. INEGI. México. (Versión electrónica)

2007. Anuario Estadístico del Estado de Veracruz de Ignacio de la Llave. INEGI. México. 250 p.

Leite, P., A. Angoa y M. Rodríguez.

2009. Emigración mexicana a Estados Unidos: balance de las últimas décadas. En: La situación Demográfica de México 2009, Consejo Nacional de Población, México, pp. 103-123.

Molina, S.

2007. Política turística en México. México: Trillas

Municipio de Catemaco.

2007. Municipio de Catemaco. (Consultada 27 de Noviembre de 2007). http://www.catemaco.gob.mx.

Municipio de San Andrés Tuxtla.

2007. Municipio de San Andrés Tuxtla. (Consultada 27 de Noviembre de 2007). http://www.sanandrestuxtla.gob.mx/index.html

Nava, T.M.

2009. Efectos de la migración en la región de Coatepec, Veracruz. En: Agricultura Sustentable volumen 6. Galdaméz G. J. F. Guevara H. L. Soto P. et al (Comp.) Universidad Autónoma de Chiapas, Sociedad Mexicana de Agricultura Sostenible A. C. México pp. 1-11. Nava, T.M.

2007. "Enfoques Teóricos de la migración rural". Conciencia Política, 13: 147-167.

Paré, L. y Lazos, E.

2003. Escuela rural y Organización comunitaria: Instituciones locales para el desarrollo y el manejo ambiental. México: UNAM-Instituto de Investigaciones Sociales / Plaza \& Janés
Piñar, Á.

2009 Desarrollo sustentable y manejo de recursos naturales en destinos turísticos de México y España: Análisis comparativo de la gestión pública y privada en municipios de las Reservas de la Biosfera de los Tuxtlas (Ver. México) y la Alpujarra (Andalucía, España). Xalapa: El Colegio de Veracruz. Subdirección de Investigación

Piñar, Á.

2007. "Desarrollo Regional y Conservación en Reservas de la Biósfera. El caso de Sierra Nevada (España)". Conciencia Política, 13: 197-222.

PNUD (Programa de las Naciones Unidas para el Desarrollo).

2005. Informe sobre Desarrollo Humano México 2004. En: www.undp.org.mx/desarrollohumano (Consultado el 15 de abril del 2008)

RECT (Red de Ecoturismo de los Tuxtlas, A.C.).

2009 Propuesta para incluir algunos temas como elementos en el diseño del taller para la elaboración de un programa educación ambiental, con base los requerimientos y especificaciones de la norma mexicana NMX-AA-133-SCFI-2006. Ms. Catemaco: RECT.

SECTUR y CESTUR (Secretaría de Turismo - Centro de Estudios Superiores en Turismo).

2007. Elementos para evaluar el impacto económico, social y ambiental del turismo de naturaleza en méxico. México: SECTUR Y CESTUR.

SECTUR (Secretaría de Turismo).

2004. Guía de apoyos federales para proyectos ecoturísticos México.

Recibido:

$24 / 08 / 10$

Reenviado:

$22 / 11 / 10$

Aceptado:

$29 / 11 / 10$

Sometido a evaluación por pares anónimos 